\title{
Vibrotactile Patterns using Sensitivity Prioritisation
}

\author{
Granit Luzhnica \\ Know-Center \\ Graz, Austria \\ gluzhnica@know-center.at
}

\author{
Eduardo Veas \\ Graz Univeristy of Technology \& \\ Know-Center \\ Graz, Austria \\ eveas@know-center.at
}

\begin{abstract}
This paper investigates sensitivity based prioritisation in the construction of tactile patterns. Our evidence is obtained by three studies using a wearable haptic display with vibrotactile motors (tactors). Haptic displays intended to transmit symbols often suffer the tradeoff between throughput and accuracy. For a symbol encoded with more than one tactor simultaneous onsets (spatial encoding) yields the highest throughput at the expense of the accuracy. Sequential onset increases accuracy at the expense of throughput. In the desire to overcome these issues, we investigate aspects of prioritisation based on sensitivity applied to the encoding of haptics patterns. First, we investigate an encoding method using mixed intensities, where different body locations are simultaneously stimulated with different vibration intensities. We investigate whether prioritising the intensity based on sensitivity improves identification accuracy when compared to simple spatial encoding. Second, we investigate whether prioritising onset based on sensitivity affects the identification of overlapped spatiotemporal patterns. A user study shows that this method significantly increases the accuracy. Furthermore, in a third study, we identify three locations on the hand that lead to an accurate recall. Thereby, we design the layout of a haptic display equipped with eight tactors, capable of encoding 36 symbols with only one or two locations per symbol.
\end{abstract}

\section{Author Keywords}

haptic feedback; tactile feedback; skin reading; stimulation; haptic display; wearable; user study; HCI

\section{ACM Classification Keywords}

H.5.2 Information Interfaces and Presentation: User Interfaces-Haptic I/O

\section{INTRODUCTION}

Wearable and mobile devices are already a part of our everyday life. They provide assistance to daily activities and enrich them with additional information collected by the sensors within

Permission to make digital or hard copies of all or part of this work for personal or classroom use is granted without fee provided that copies are not made or distributed for profit or commercial advantage and that copies bear this notice and the full citation on the first page. Copyrights for components of this work owned by others than the author(s) must be honored. Abstracting with credit is permitted. To copy otherwise, or republish, to post on servers or to redistribute to lists, requires prior specific permission and/or a fee. Request permissions from permissions@acm.org.

ISWC '17, September 11-15, 2017, Maui, HI, USA

(C) 2017 Copyright is held by the owner/author(s). Publication rights licensed to

ACM.

ACM 978-1-4503-5188-1/17/09...\$15.00

https://doi.org/10.1145/3123021.3123032 them. The primary feedback modalities of mobiles and wearables are visual and auditory. As such, they compete for visual and auditory attention and distract the user from important tasks. Alternative display modalities, such as tactile displays, can reduce demands on the predominant visual display, but are largely under-utilised [2].

With the proliferation of wearables, devices with vibrotactile capabilities are accessible to a substantial number of end users. Currently, the primary utilisation of vibrotactile feedback is to provide additional support to visual interaction. Nevertheless, haptic feedback can of transmitting rich information without the need to perceive it through auditory or visual channels. There is already extensive research exploring capabilities of haptic feedback for different purposes, such as skin reading [8, 19], navigation aids [5, 4], presenting visual information to car drivers [26], assistive systems in medical surgery [14], enabling haptic experiences in story telling [34], and enhancing experiences on virtual reality $[3,17]$, augmented reality $[13$, $31]$, and multi-media systems [22, 23, 28].

The primary focus of our research is offering methods for haptic displays to encode a vocabulary of symbols that can be combined into complex messages. Such methods can be used, for example, to perceive natural language messages encoded in vibrotactile patterns $[8,19]$. The proposed methods can benefit displays with broad application possibilities. Users would be able to receive and understand messages and notifications from the mobile phone without even having to get it out of the pocket. Deaf users would be able to use speech to text (captured by a smartphone) and text to tactile to fully understand other persons talking to them. Workers in factories could receive work instructions while working without deviating visual and auditory attention from their work. Several other scenarios can benefit from general purpose wearable displays and, most importantly, the barriers of technology (wireless communication, batteries, integration to fabrics) for making such haptic displays fully wearable have been overcome. A critical aspect of a haptic display is encoding of information. One major challenge when encoding a vocabulary of symbols in a small number of haptic actuators is maintaining a high throughput and accuracy. As the number of actuators encoding a symbol increases, they suffer from a masking effect [19, 24, $21]$, whereby the stimulation of some tactors is not felt, and the pattern is confused with another, thereby reducing accuracy. 
This work unveils fine-grained details of vibrotactile patterns to increase the accuracy of perceiving such patterns and shorten their duration. The main contribution of our work is investigating the effects of sensitivity based prioritisation in the encoding of tactile patterns. We investigate the effect of using different stimulation intensities in a single pattern. Additionally, we present a detailed investigation of whether prioritising the activation of vibrotactile tactors has an effect on the correct perception and identification of locations. The patterns use an overlapped spatiotemporal (OST) encoding where most of the activation time is shared between tactors. The prioritisation is done based on the sensitivity of the locations, and we investigate the order of onset based on more sensitive locations first or least sensitive location first. Moreover, we investigate the comparative sensitivity of locations in hand (other than the fingers) and apply the results in the design of a haptic display. Hereby, our overall contribution lies in investigating sensitivity based prioritisation in encoding haptics patterns, backed by empirical evidence obtained with a wearable display using vibrotactile motors.

\section{MOTIVATION}

The methods proposed in this paper are intended to encode large vocabularies in vibrotactile patterns that can be combined to form complex messages. We intend to do so with a maximum accuracy and throughput. Hereby, we investigate the effect of sensitivity based prioritisation of stimulus.

Our haptics display consists of vibrotactile motors (tactors) operating at around $220 \mathrm{~Hz}$. Hereby, stimulation is mostly occurring in the cutaneous subsystem, which is sensitive in the range of $20-1000 \mathrm{~Hz}$, with maximum sensitivity around $250 \mathrm{~Hz}[10,9]$. Cutaneous receptors determine the spatial and temporal resolving capacity of the skin (spatial and temporal acuity) and are spread with different densities in the body. Spatial acuity is characterised by the two point discrimination - the minimum distance required for two spatial stimuli to be discriminated, which for fingers is around $5 \mathrm{~mm}[14,16]$, about $1 \mathrm{~cm}$ for palm and $4 \mathrm{~cm}$ for forearm [16]. Temporal acuity studies indicate that people can discriminate between successive taps on the skin with a gap of $5 \mathrm{~ms}$ [11]. From the characteristics of the cutaneous system and vibrotactile actuators, the symbols of a vocabulary can be encoded with variations in amplitude, frequency, duration and body location. For example, Geldard [8] used five tactors placed on the chest to encode 45 symbols (letters, numbers and most frequent short words) in combinations of loci, amplitude and duration. Onset specifies how tactors are activated when using two or more tactors to encode a symbol. Spatial encoding means that all tactors in a symbol are onset concurrently. Spatial encoding suffers from a masking effect [21, 19, 24], where the simultaneous activation of tactors results on the higher sensitive locations masking the perception of lower sensitive locations. On the hand, the sensitivity decreases from the index finger towards the little finger $[6,30,12]$ : the index finger is more sensitive than the middle, ring, and pinky finger. To compensate for the masking effect, we propose to prioritise stimulation of the least sensitive location.
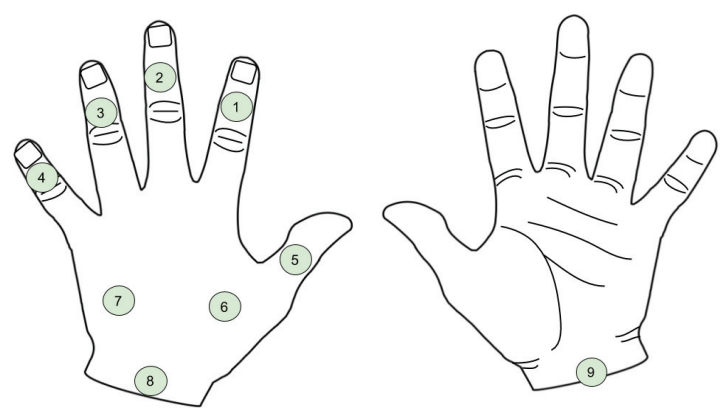

Figure 1: The haptic display investigated in this paper. RQ1 and RQ2 are addressed with studies using the tactors on the fingers $(1-4)$, and RQ3 uses the back of the hand and wrist locations $(6-9)$

Sensitivity prioritised intensity of stimulation. Our first assumption is that stimulating less sensitive locations with higher intensity yields a higher accuracy in recognising a pattern. In other words, different intensities are used for each tactor, with the tactor on a more sensitive location being stimulated with a lower intensity than the tactor in a less sensitive location. With this encoding, the transmission time remains constant. In this context, our first research question (RQ1) is:

RQ1. Does the simultaneous activation of tactors with different intensities result in higher identification accuracy compared to using the same intensity in all tactors?

Another form of encoding is spatiotemporal encoding, whereby symbols are activated one at a time, in sequence [21]. Thus, preventing the masking effect. But, such encoding yields a higher duration for each pattern and decreases throughput as a consequence. Luzhnica et al. used an overlapped spatiotemporal (OST) encoding, where onset occurs in sequence after a time gap for each tactor after the first one [19]. They used a time gap of $10 \mathrm{~ms}$, twice the minimum temporal difference of $5 \mathrm{~ms}$ [11]. Luzhnica et al. did not investigate the effect of onset prioritisation.

Sensitivity prioritised onset of stimulation. Our second assumption is that a sensitivity prioritised onset of stimulation using OST leads to higher recognition accuracy. In this case, the tactors in a pattern are activated in sequence after a gap. The sequence of activation is given by the sensitivity of skin in the tactor location. All tactors remain activated for the duration of the pattern. The second research question is:

RQ2. Does the prioritisation of activation of tactors have an effect on the accuracy of identification of each tactor when using an overlapping spatiotemporal (OST) encoding? How should we prioritise, least sensitive to most sensitive locations or vice-versa?

When using OST to encode a vocabulary, it was found that encoding patterns with more than two tactors resulted in significantly lower accuracy than the cases with one and two tactors [19]. The techniques in this paper concentrate on twotactor patterns. Thus, to increase the size of the vocabulary that can be encoded, it becomes necessary to add tactors in different locations. The number of symbols that can be encoded 
with one or two tactors is:

$$
n=\left(\begin{array}{c}
m \\
2
\end{array}\right)+m=\frac{m(m-1)}{2}+m=\frac{m(m+1)}{2}
$$

where $m$ represents the number of tactors in the haptic display. A display with $m=7, n=28$ can encode the entire English alphabet plus two other characters (e.g. space and period). With $m=8, n=36$ and with $m=9, n=45$ it would be sufficient to also encode most of the punctuations and symbols. Tactors have been successfully used on the fingers used for such tasks [19], we investigate the effect of using tactors in less sensitive areas of the hand. Our third research question is: RQ3. Can stimulation with high throughput and accuracy be achieved in less sensitive parts of the hand?

\section{RELATED WORK}

Early attempts to encode information through passive tactile date from 1924, where Gault [7] used a piezoelectric unit to convert entire recorded speech to touch. Similarly, Kirman [15] used a $15 \times 15$ vibrator matrix on the palm to teach six participants to differentiate between the patterns of 15 different words. Other researchers attempted to utilise a visually oriented approach where the low-resolution image of the object is projected to an array of stimulators. For instance, White [32] transformed images captured from a video feed to a $20 \times 20$ vibrotactile display placed on the back. After training, participants were able to distinguish simple shapes like circle, square and triangle. By following this approach, Bliss [1] developed the first commercial device capable of capturing text from the video feed and then imprinting each letter on the finger with a $6 \times 24$ matrix of vibrators.

A more successful approach of transmitting information through haptics was provided by Geldard [8] in 1967. The device was named Vibratese, and it used five tactors placed on the chest to encode 45 symbols (letters, numbers and most frequent short words). The author reported that after 65 hours of training one participant was able to understand $38 \mathrm{wpm}$ (words per minute). More recently, Luzhnica [19] followed a different encoding scheme using only the location of tactors to encode 26 letters of English alphabet. The authors used six tactors on the back of the hand and were able to train users to perceive letters, words and phrases within only five hours.

A critical aspect of tactile displays is how they encode the information. Encoding needs to provide patterns that are discriminative. But it also needs to deliver them as fast as possible. Typically a combination of variations in amplitude [27, 29, 33], frequency [27, 29, 33], duration [9, 8] and body locations [8, $33,20,25]$ have been used. For instance, Geldard [8] in his Vibratese work used five locations, a variation of three durations and three intensities to encode the desired symbols. Recently, Novich [21] showed that spatiotemporal encoding, where tactors in a pattern are turned on and off sequentially one after the other, results in significantly better discrimination than the spatially encoded patterns where all tactors in a pattern onset simultaneously. Liao [18] utilised such a spatiotemporal encoding to encode symbols on the wrist. Although such encoding works well $[18,21]$ in terms of being identified by participants, it is many times slower than the spatial encoding.
Luzhnica [19] used a prioritised overlapping spatiotemporal encoding where tactors are activated in sequence after a gap, and they stay on until the pattern is finished. This method resulted in better recognition accuracy than spatial encoding, and it is faster than spatiotemporal encoding, as tactors share most of the activated time.

\section{METHODOLOGY}

We address our research questions with three user studies. In the first study (RQ1), we compose patterns that differ on vibration intensity for each tactor and investigate the effects of such variations. In the second user study (RQ2), we compose overlapping spatiotemporal patterns consisting of one or two tactors. Patterns differ on the gap between the activation of tactors and their order. Sensitivity prioritisation guides the onset of tactors in a pattern. We analyse its effects combined with gap duration. In both studies, we use only four tactors as we aim to keep participants interested and at the same time gather enough data for statistical analysis. We concentrate on the fingers as locations, because of their known sensitivity order [6, 30, 12].

In a third user study (RQ3), we add four tactors to the display design. We add tactors in such a way that everything could be placed inside a fingerless glove. No tactor is placed on the palm, to avoid interference with everyday interactions. As shown in Figure 1, two of tactors (6 and 7) are placed on the back of the hand, at acceptable discrimination distances as given by the cutaneous sensitivity of the hand. Additionally, there are two tactors near the wrist, one on the back and one on the front of the hand. The primary concern about this design is whether combinations of tactors 6,7 and 8 can be used within the same pattern, considering the small distance between them. Additionally, as the tactor 9 is on the opposite side of tactor 8 , would their combination be recognisable as well? Combining fingers with a single tactor on the back of the hand was tested by Luzhnica et al. [19]. Their setup had a similar distance between the hand and finger tactors which is assuring that the distance between hand motors and fingers is enough to avoid masking. Thus, we do not study patterns combining finger tactors $(1-5)$ but hand tactors $(6-9)$ to identify whether such positions are suitable for our task.

In all studies, we investigate how accuracy is affected by stimulation method, prioritisation, and locations of tactors. Accuracy is defined to be a binary variable set to true if the participant identifies all the locations/tactors that compose the pattern and false otherwise. We use chi-square to determine whether there is a significant difference in accuracy between two groups. When comparing more than two groups, we use Bonferroni correction to determine the significance threshold.

\section{STUDY1: SENSITIVITY PRIORITISED INTENSITY}

This study investigates the use of different intensity of stimulation according to the sensitivity of the location. Our assumption is that using different intensities on both tactors with simultaneous onset; the identification accuracy will increase compared to using the same intensity (spatial). 


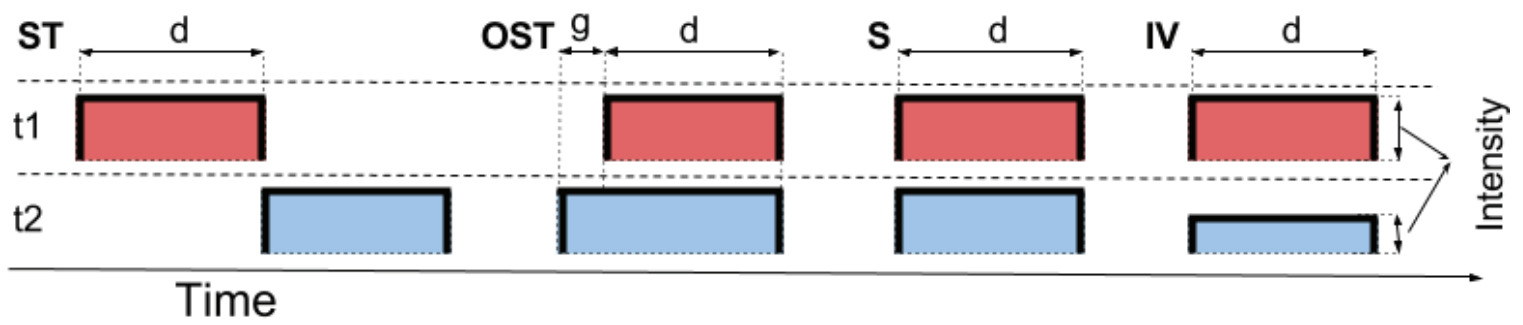

Figure 2: Pattern types composed of two tactors/locations: spatiotemporal (ST), overlapping spatiotemporal (OST), spatial (S) and intensity varying (IV). Base duration $(d)$ represents the activation time of a tactor ( $\mathrm{t} 1$ and $\mathrm{t} 2$ ). The gap between the activation of tactors is denoted by $g$. The height or rectangle represents the intensity of the vibration.

\begin{tabular}{r||c|c|c|c} 
PT & PWD $_{1}$ & PWD $_{2}$ & $\mathrm{~g}(\mathrm{~ms})$ & $\mathrm{d}(\mathrm{ms})$ \\
\hline S & 1 & 1 & 0 & 100 \\
\hline IV1 & 1 & 0.75 & 0 & 100 \\
IV2 & 1 & 0.50 & 0 & 100 \\
\hline OST1 & 1 & 1 & 10 & 100 \\
OST2 & 1 & 1 & 20 & 100
\end{tabular}

Table 1: Pattern types (PT) used on the user studies. PWD $_{1}$ and $\mathrm{PWD}_{1}$ represent the duty cycles (vibration intensities) of the first and second tactor of the pattern. The base duration is denoted by $d$ and the gap between activation by $g$.

\section{Apparatus}

Our device consisted of an Arduino Due board which controls $3.4 \mathrm{~mm}$ vibrotactile motors of type ROB-08449 (Voltage range: $2.3 \mathrm{~V} \sim 3.6 \mathrm{~V}$; Amplitude vibration: $0.8 \mathrm{G}$ ).

\section{Procedure}

We used four tactors (see tactors $1-4$ ) as shown in Figures 1 and 3 . For each permutation of the tactors, a set of patterns with two tactors is generated for spatial (S) and two (IV1, IV2) types of intensity varying patterns (IV) where one of the tactors is activated with a lower intensity than the other one (see Table 1 and Figure 2). The spatial encoding (S) uses the same intensity on both tactors, and it will serve as a baseline to compare with other pattern types. The two types of IV patterns differ in the intensity of vibration used on the second tactor (see Table 1). Thus, in total, we used three sets of patterns ( $\mathrm{S}$, IV1, IV2). Figure 2 illustrates the patterns used in the study. Note that as we use an Arduino device to control our tactors, we are technically unable to set the intensity of the tactors (Arduino devices do not have analogue output). Nevertheless, the effect of a lower intensity is achieved by setting a lower duty cycle of pulse width modulation (PWD). A duty cycle of 1 produces the highest vibration intensity.

Since for each permutation of tactors a pattern is generated, for spatial (S) patterns, each pattern was included twice on the set (as the pattern with tactors $1-2$ is the same as $2-1$ ). In the case of IV type (IV1 and IV2), for every two tactors, two patterns with an opposite order of activation are included (e.g. $1-2$, where 1 is activated first and then 2 after a gap and $2-1$, where the order is reversed). Additionally, each set included a pattern with a single tactor (with max intensity) for each of the available tactors. In total each of the five sets included 16
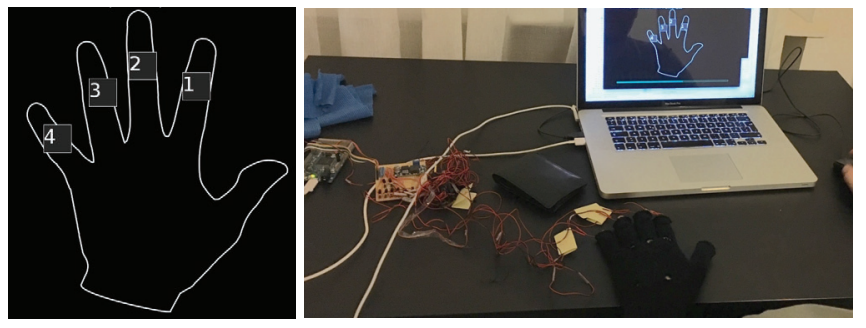

Figure 3: The user interface of the first user study (left) and a picture of a participant during this study (right).

patterns (12 with two tactors and 4 with one tactor). The main reason to include single tactor patterns is to prevent the cases where users feel only one tactor, but being aware that there are only two-tactor patterns, motivates them to guess one they did not feel. Each participant was tested twice for each three sets (S, IV1 and IV2) of patterns. Therefore each participant was tested for $72(2 \times 3 \times 12)$ probes with two tactors and 24 $(2 \times 3 \times 4)$ probes with single tactor.

The entire experiment was controlled by a Python-based application, which for each pattern in the probes, stimulated participants in a randomised order and then asked them to select the tactors in the user interface, by selecting the rectangles representing tactors (see Figure 3) using the mouse. Participants could repeat the stimulation once if they were distracted while the stimulation was applied (e.g. if they were making a comment or a question).

\section{Participants}

Eleven participants (six male, five female) took part in the study. All of them were right handed, and we used the left hand for stimulation. The right hand was used to operate the mouse.

\section{Results}

Initially, we introduce a new variable called order for pattern types IV1, IV2. We define the pattern to be ordered if the index of the first tactor is smaller than the index of the second tactor. Otherwise, the order is reversed. If the pattern is ordered, then it is prioritised to stimulate the higher sensitive place with higher intensity than the lower sensitive place. If it is reversed, the least sensitive place is stimulated with higher intensity. As presented in Figure 4, both ordered, and inverse variants of 

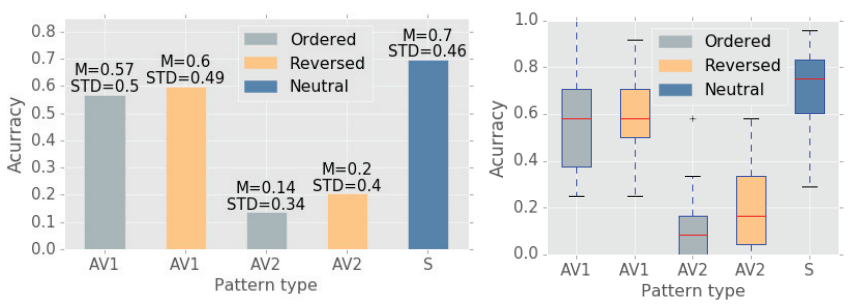

Figure 4: Correct identification of patterns for each pattern types (left) used during the first study. The box plot (right) presents the results averaged per user.
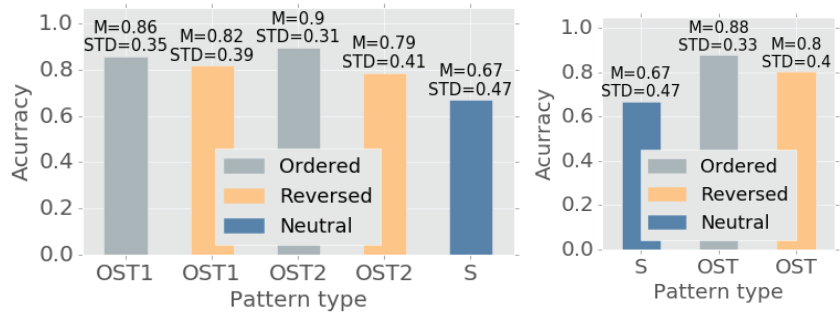

Figure 5: Correct identification of patterns for each pattern types (left) used during the second user study.

IV1 and IV2 result in worse accuracy than the spatial patterns (S). Chi-square comparisons reveal:

1. S vs IV1 ordered: $\chi^{2}(2, N=396)=5.89, p=0.015$,

2. S vs IV1 reversed: $\chi^{2}(2, N=396)=3.4, p=0.065$,

3. S vs IV2 ordered: $\chi^{2}(2, N=396)=108.44, p=0.0$,

4. $\mathrm{S}$ vs IV2 reversed: $\chi^{2}(2, N=396)=83.76, p=0.0$

For IV1, when comparing ordered vs reversed, a chisquare comparison reveals the differences are not significant $\chi^{2}(2, N=264)=0.14, p=0.71$. Similarly, for IV2 the changes between ordered and reversed are not significant $\chi^{2}(2, N=264)=1.71, p=0.19$. When comparing $\mathrm{S}$ with IV1 (both ordered and reversed) the changes are significant $\chi^{2}(2, N=528)=6.91, p<0.01$. Also, for S and IV2 $\chi^{2}(2, N=528)=146.85, p=0.0$ the changes are significant. Similarly, the changes between IV1 and IV2 are significant $\chi^{2}(2, N=528)=94.07, p=0.0$.

\section{STUDY2: SENSITIVITY PRIORITISED ONSET}

This study aims to investigate the effect of prioritisation on the tactors onset when using OST stimulation. We assume that prioritising onset based on the sensitivity of location yields a better accuracy in identifying the locations of stimulus.

\section{Procedure}

We create a set of spatial encoding patterns (S) and two (OST1, OST2) overlapping spatiotemporal (OST) types patterns where a gap between activation of tactors is used (see Table 1 and Figure 2). The rest of the procedure was identical to the first study. Each participant was tested twice for each three sets (S, OST1, OST2) of patterns. Therefore each participant was tested for $72(2 \times 3 \times 12)$ trials with two tactors and 24 $(2 \times 3 \times 4)$ trials with a single tactor.
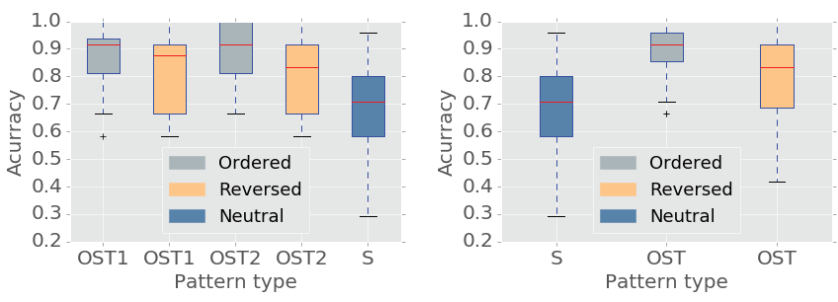

Figure 6: Correct identification of patterns for each pattern types (left) used during the second study. The results are averaged per user.

\section{Participants}

Twenty participants (eleven male, nine female) took part in the study.

\section{Results}

We introduce order as a variable for pattern types OST1, OST2. If the pattern is ordered, then the location with higher sensitivity is stimulated first, and if it is reversed, then the lowest sensitivity location is prioritised. The average identification accuracies of ordered, reversed and neutral patterns are presented in Figure 5. Additionally a boxplot of averages for each user is presented in Figure 6. The Figures ( 5 and 6) reveal that ordered OST performs better than reversed (for both OST1 and OST2) and all combinations of OST perform better than S. Nevertheless, for determining significance we will use chi-square test.

Comparing S with ordered and reversed of OST (combined OST1 and OST2) reveals that changes between $\mathrm{S}$ and both ordered and reversed OST are significant $\chi^{2}(2, N=960)=$ $57.56, p=0.0$; respectively $\chi^{2}(2, N=960)=24.54, p=0.0$. Additionally also the changes between ordered and reversed OST are significant $\chi^{2}(2, N=960)=7.23, p=0.0072$. On the other hand, all combinations of OST and ordering performed significantly better than baseline $\mathrm{S}^{1}$ :

1. OST1 ordered vs $\mathrm{S}: \chi^{2}(2, N=720)=27.28, p=0.0$,

2. OST1 reversed vs $\mathrm{S}: \chi^{2}(2, N=720)=19.92, p=0.0$,

3. OST2 ordered vs $\mathrm{S}: \chi^{2}(2, N=720)=42.64, p=0.0$ and

4. OST2 reversed vs $\mathrm{S}: \chi^{2}(2, N=720)=11.24, p=0.0008$

Additionally, the baseline (S) seems to be performing significantly worse than both OST1 $\chi^{2}(2, N=960)=38.33, p=$ 0.0 ; and OST2 $\chi^{2}(2, N=960)=39.39, p=0.0$. When comparing the ordering within OST1 and OST2, for OST1 the differences do not seem to be significant between ordered and reversed $\chi^{2}(2, N=480)=0.4, p=0.527$; whereas for OST2 the changes are significant $\chi^{2}(2, N=480)=9.31, p=0.002$.

Interestingly, the differences between OST1 and OST2 do not seem to be significant $\chi^{2}(2, N=960)=0.1, p=0.75$. Also the differences between ordered OST1 and ordered OST2 are not significant $\chi^{2}(1, N=480)=0.0, p=1.0$. Similarly, the differences between reversed OST1 and reversed OST2 are not significant $\chi^{2}(1, N=480)=0.0, p=1.0$.

\footnotetext{
1 as significance we use a threshold of $\alpha=0.0125$ according to following Bonferroni correction
} 


\begin{tabular}{c|c|c|c|c|c|c|c|c|c|c|c} 
& \multicolumn{9}{c}{$\mathbf{S}$} & \multicolumn{9}{c}{ OST1 } & \multicolumn{3}{c}{ OST2 } \\
\hline $\mathbf{t 1 / t 2}$ & $\mathbf{7}$ & $\mathbf{8}$ & $\mathbf{9}$ & $\mathbf{6}$ & $\mathbf{7}$ & $\mathbf{8}$ & $\mathbf{9}$ & $\mathbf{6}$ & $\mathbf{7}$ & $\mathbf{8}$ & $\mathbf{9}$ \\
\hline $\mathbf{6}$ & $.79(.41)$ & $.29(.46)$ & $.70(.46)$ & & $.94(.24)$ & $.62(.49)$ & $.96(.20)$ & & $.85(.36)$ & $.77(.42)$ & $.94(.24)$ \\
\hline $\mathbf{7}$ & & $.32(.47)$ & $.67(.47)$ & $.96(.20)$ & & $.75(.44)$ & $.83(.38)$ & $.92(.28)$ & & $.88(.33)$ & $.98(.14)$ \\
\hline $\mathbf{8}$ & & & $.43(.50)$ & $.50(.51)$ & $.50(.51)$ & & $.69(.47)$ & $.62(.49)$ & $.65(.48)$ & & $.60(.49)$ \\
\hline $\mathbf{9}$ & & & & $.85(.36)$ & $.85(.36)$ & $.65(.48)$ & & $.81(.39)$ & $.90(.31)$ & $.71(.46)$ &
\end{tabular}

Table 2: Results of the second study for each combination of two tactors. The row defines the first activated tactor whereas the column defines the second. In the case of S both tactors are activated in parallel, therefore, the results are displayed together. Color coding: $\bigcirc$ - spatial, $\bigcirc$ - ordered (OST), $\bigcirc$ - reversed (OST).

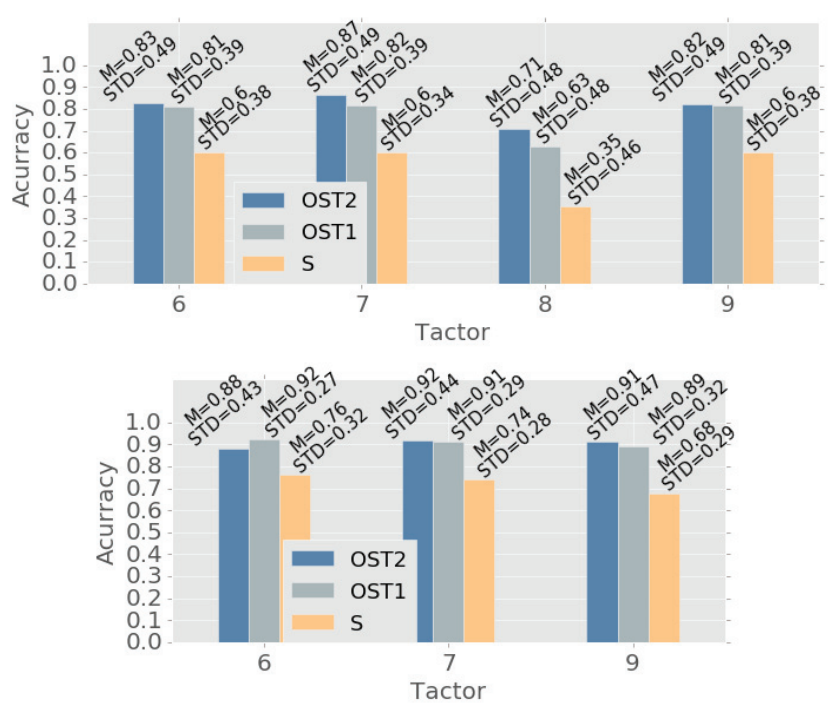

Figure 7: Correct identification of patterns for each pattern that involves the tactor. Please note that each pattern is included in two categories as it contains two tactors. Therefore, when removing tactor 8 , the accuracies increase in other groups.

\begin{tabular}{c|c|c|c|c} 
Tactor & 6 & 7 & 8 & 9 \\
\hline Accuracy & $.99(.08)$ & $.99(.12)$ & $.90(.30)$ & $.99(.12)$
\end{tabular}

Table 3: Average correct identification of patterns composed of only one tactor.

\section{STUDY3: ADDING MORE TACTORS ON THE HAND}

The third study investigates how well OST patterns with two tactors can be recognised on less sensitive parts of the hand.

\section{Procedure}

Three tactors were placed on the back of the hand (one of them near the wrist) and one on the palm side near the wrist. The exact positions are given on Figure 1 (tactors 6-9) of the new design. Apart from the position of tactors, the rest of the study was organised in the same manner as the second study. Three sets of probes (S, OST1 and OST2) were used in a randomised order to test the participants for identification. In total each user was tested for for $108(3 \times 3 \times 12)$ probes with two tactors and $36(3 \times 3 \times 4)$ probes with one tactors.

\section{Participants}

In this study participated 15 people (seven male, eight female).

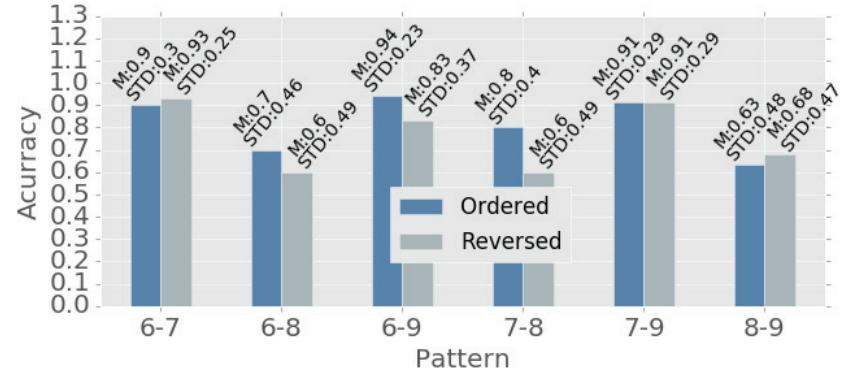

Figure 8: Correct identification of patterns for each pattern that involves two tactors (only for OST patterns).

\section{Results}

The accuracies for each combination of tactors are presented in Table 2. The table shows that all the patterns that involve tactor 8 perform worse than the others. To elaborate on this result, in Figure 7 we illustrate the accuracies of patterns grouped by tactors that they contain. On the top, we visualise the accuracies of all patterns, whereas, on the bottom, we visualise only ones that do not involve tactor 8 . Note that groups are not exclusive as each pattern belongs to two groups (e.g., patterns 6-7 belongs to both groups of tactor 6 and tactor 7) and hence inaccuracies of patterns involving tactor 8 affect other groups as well. Here, when comparing pairwise, each group is statistically significant compared to group 8 ( 6 vs 8 : $\chi^{2}(2, N=1728)=62.55, p=0.0 ; 7$ vs $8: \chi^{2}(2, N=1728)=$ $73.15, p=0.0$; and 9 vs $\left.8: \chi^{2}(2, N=1728)=64.25, p=0.0\right)$.

In addition, we present the accuracies of patterns composed of only one tactor in Table 3. When looking at the comparison between acurracies for patterns with one tactor only, the differences between all other tactors and tactor 8 are significant $\left(6\right.$ c $8: \chi^{2}(2, N=288)=10.13, p=0.0015 ; 7$ vs 8 : $\chi^{2}(2, N=288)=8.01, p=0.0047$; and 9 vs $8: \chi^{2}(2, N=$ $288)=8.01, p=0.0047)$, whereas the differences between tactors 6, 7 and 9 are not. Both comparisons (Figure 7 and Table 3) point out that the location for tactor 8 is not a good choice for a haptic display.

Following the second study where onset prioritisation resulted in higher accuracy, we would like to define the order of activation for positions on hand as well. While for the first and second studies the sensitivities are well known and studied [6, $30,12]$, for the positions chosen in this study, to the best of our knowledge, there is no evidence comparing their sensitivities. For this, in Figure 8, we present the average accuracies 
for all combinations of tactors including the order for OST pattern types (both OST1 and OST2). Comparing each of them (e.g 6-7 vs 7-6) reveals that the differences between 6-9 vs 9-6 $\left(\chi^{2}(2, N=192)=5.35, p=0.0208\right)$ and $7-8$ vs $8-7$ $\left(\chi^{2}(2, N=192)=11.84, p=0.0006\right)$ are significant.

Based on this evidence, the priorities of stimulation for tactors 6 and 7 would be higher than 9. Between 6 and 7, we would prioritise 7 just because of the average accuracy, but either way, it would not make a major difference. Whereas for tactor 8 , we would remove it from our design as long as we would not need to encode a vocabulary with more than 36 symbols.

\section{DISCUSSION}

In the first study, we investigated whether by varying the intensity of vibration we can provide an encoding which results in a better accuracy than the baseline (S) without using any gap in between the activation of tactors. At least with intensities that we investigated (which were controlled by a duty cycle of PWD), such an encoding did not even achieve the same accuracy as S, let alone exceed it. Nevertheless, we do not immediately discourage other researchers to investigate the same technique with actuators that offer a more accurate intensity control. It is entirely possible that by tuning the intensities (investigating other levels of intensities), this might bring better results. Within the frame and settings of our study, such an encoding technique did not prove to be useful.

Our second study reveals that participants identified the stimuli significantly better using OST than S. Participants also performed significantly better when the order of tactors was from smallest to the highest index (for the OST). Since that order is the exact order of sensitivity of the locations $[6,30,12]$, this suggests that prioritising the onset of tactors based on sensitivity in OST encoding significantly increases the accuracy of identification of patterns. Surprisingly, increase in accuracy was achieved by prioritising the most sensitive locations first, which is the opposite of what was assumed to be the case in previous research [19]. Intuitively, one would expect that by prioritising the least sensitive location while the more sensitive one is not active (gap), users would perceive it. Later when both tactors are activated, even if the least sensitive place is masked by the more sensitive, participants already are aware of its simulation (during the gap). Although this encoding is significantly better than the baseline, the opposite, prioritising the most sensitive location, works significantly better. Perhaps exactly the kick of the second activation is much more efficient mechanism against masking. It is also interesting that the gap (10 ms vs. $20 \mathrm{~ms}$ ) used between activation of tactors in OST did not have a significant effect on identification accuracy. In our settings (base duration of $100 \mathrm{~ms}$ ), $20 \mathrm{~ms}$ gap increases the total duration of patterns for $9 \%(110 \mathrm{~ms} v \mathrm{vs} .120 \mathrm{~ms})$ over the $10 \mathrm{~ms}$ gap. Despite the overhead which will affect the throughput when encoding symbols, it still did not result in a significant gain in accuracy.

In the third study, we added four tactors on the hand, and we tested all combinations of patterns composed by one and two tactors. The results revealed that tactor 8 is comparably poor for haptic stimulation as patterns that contain it were identified significantly worse than patterns that do not. Other locations seem to provide comparably good accuracy with both OST encoding types. Please note that even though accuracy is not $100 \%$, they are still a good fit for a haptic display as there is some learning effect to it. For instance, in [19], participants after some training time performed much better in identifying the symbol associated with the pattern $(98 \%$ accurate within one hour of training) than they performed in a pre-study where they were asked identify the location of stimulus (83\% on the hand for patterns with two tactors). This suggests that as users are exposed more to the stimulus, they can identify more accurately the stimulus. Considering the results of the third study, our final design of the glove based haptic display is composed on eight tactors. Seven $(1-7$ in Figure 1) of them placed on the back of the hand whereas one is placed on the wrist of the palm side of the hand (9 in Figure 1). With eight tactors, we would be able to encode 36 different symbols using prioritised OST, which is enough for the entire English alphabet and most important punctuations.

\section{CONCLUSION}

In this paper, we investigate detailed aspects of hand based tactile display for encoding large vocabularies consisting of 36 symbols. We present results of investigating methods that use sensitivity based prioritisation in encoding, which ensures high throughput and accuracy.

First, we show that using different vibration intensities between tactors does not contribute to a higher accuracy (even when they are prioritised by sensitivity) than the baseline spatial encoding where the intensities are kept constant for both tactors. Next, we examine whether the order of activation of tactors in an overlapping spatiotemporal stimulation has an effect in correctly identifying the stimulus. Our results suggest that prioritising the activation of tactors based on highest sensitive place towards lowest significantly increases the accuracy. Our results are surprising and exactly the opposite of what authors in [19] assumed. Prioritising the tactors suggest, will contribute to an increase in perception accuracy.

Moreover, we extended our investigation on sensitivity to additional hand locations [19]. We experimented with four additional locations and kept three for a final design with eight tactors. This paper presented design guidelines for sensitivity based prioritisation in encoding haptics patterns. The guidelines are backed by empirical evidence obtained with a wearable display using tactors. It is our hope that the evidence and guidelines defined in this research paper will find their way in the design of wearable haptics displays in the future.

\section{ACKNOWLEDGMENTS}

This work is funded by MoreGrasp (H2020-ICT-2014-1 643955). The KnowCenter is funded within the Austrian COMET Program - Competence Centers for Excellent Technologies - under the auspices of the Austrian Federal Ministry of Transport, Innovation and Technology, the Austrian Federal Ministry of Economy, Family and Youth and by the State of Styria. COMET is managed by the Austrian Research Promotion Agency FFG.

\section{REFERENCES}

1. J. C. Bliss, M. H. Katcher, C. H. Rogers, and R. P. Shepard. 1970. Optical-to-Tactile Image Conversion for the Blind. IEEE Transactions 
on Man-Machine Systems 11, 1 (March 1970), 58-65. DOI : http://dx.doi.org/10.1109/TMMS . 1970.299963

2. Stephen Brewster and Lorna M. Brown. 2004. Tactons: Structured Tactile Messages for Non-visual Information Display. In Proceedings of the Fifth Conference on Australasian User Interface (AUIC). 15-23. http://dl . acm.org/citation. cfm?id=976310.976313

3. Jurgen Broeren, Martin Rydmark, and Katharina Stibrant Sunnerhagen. 2004. Virtual reality and haptics as a training device for movement rehabilitation after stroke: A single-case study1. Archives of Physical Medicine and Rehabilitation 85, 8 (2004), 1247 - 1250. DOI : http://dx.doi .org/10.1016/j . apmr.2003.09.020

4. de Jesus Oliveira and Anderson Maciel. 2014a. Assessment of Tactile Languages as Navigation Aid in 3D Environments. In EUROHAPTICS, 2014 .

5. de Jesus Oliveira and Anderson Maciel. 2014b. Introducing the Modifier Tactile Pattern for Vibrotactile Communication. In EUROHAPTICS, 2014.

6. Robert O. Duncan and Geoffrey M. Boynton. 2007. Tactile Hyperacuity Thresholds Correlate with Finger Maps in Primary Somatosensory Cortex (S1). Cerebral Cortex 17, 12 (2007), 2878. D0I : http://dx.doi.org/10.1093/cercor/bhm015

7. Robert H Gault. 1924. Progress in experiments on tactual interpretation of oral speech. The Journal of Abnormal Psychology and Social Psychology 19, 2 (1924), 155.

8. Frank A. Geldard. 1957. Adventures in tactile literacy. American Psychologist 12, 3 (1957), 115-124.

9. Eric Gunther. 2001. Skinscape: A tool for composition in the tactile modality. Ph.D. Dissertation. Massachusetts Institute of Technology.

10. Eric Gunther, Glorianna Davenport, and Sile O'Modhrain. 2002. Cutaneous Grooves: Composing for the Sense of Touch. In Proceedings of the 2002 Conference on New Interfaces for Musical Expression (NIME '02). National University of Singapore, 1-6. http://dl . acm.org/citation. cfm?id=1085171. 1085181

11. Morton A Heller and William Schiff. 2013. The psychology of touch. Psychology Press.

12. Eve E. Hoggan, Sohail Anwar, and Stephen A. Brewster. 2007. Mobile Multi-actuator Tactile Displays. 22-33 pages. DOI : http://dx.doi .org/10.1007/978-3-540-76702-2_4

13. Wolfgang Hürst, Nina Rosa, and Jean-Paul van Bommel. 2016. Vibrotactile Experiences for Augmented Reality. In Proceedings of the 2016 ACM on Multimedia Conference (MM '16). ACM, New York, NY, USA, 744-745. DOI: http://dx.doi.org/10.1145/2964284.2973830

14. Thorsten A. Kern. 2009. Engineering Haptic Devices: A Beginner's Guide for Engineers (1st ed.). Springer Publishing Company, Incorporated.

15. Jacob H. Kirman. 1974. Tactile perception of computerâĂ ̌̌derived formant patterns from voiced speech. The Journal of the Acoustical Society of America 55, 1 (1974).

16. S. J. Lederman and R. L. Klatzky. 2009. Haptic perception: A tutorial. Attention, Perception, \& Psychophysics 71, 7 (2009). DOI: http://dx.doi.org/10.3758/APP.71.7.1439

17. G Michael Jr. Lemole, P Pat Banerjee, Cristian Luciano, Sergey Neckrysh, and Fady T Charbel. 2007. Virtual reality in neurosurgical education: part-task ventriculostomy simulation with dynamic visual and haptic feedback. Neurosurgery 61, 1 (2007). http: //journals. Iww . com/neurosurgery/Fulltext/2007/07000/VIRTUAL

18. Yi-Chi Liao, Yi-Ling Chen, Jo-Yu Lo, Rong-Hao Liang, Liwei Chan, and Bing-Yu Chen. 2016. EdgeVib: Effective Alphanumeric Character Output Using a Wrist-Worn Tactile Display. In Proceedings of the 29th Annual Symposium on User Interface Software and Technology (UIST '16). ACM, New York, NY, USA, 595-601. DOI : http://dx.doi .org/10.1145/2984511.2984522
19. Granit Luzhnica, Eduardo Veas, and Viktoria Pammer. 2016. Skin Reading: Encoding Text in a 6-channel Haptic Display. In Proceedings of the 2016 ACM International Symposium on Wearable Computers (ISWC '16). ACM, New York, NY, USA, 148-155. DOI : http://dx.doi.org/10.1145/2971763.2971769

20. Hugo Nicolau, João Guerreiro, Tiago Guerreiro, and Luís Carriço. 2013. UbiBraille: Designing and Evaluating a Vibrotactile Braille-reading Device. In Proceedings of the 15th International ACM SIGACCESS Conference on Computers and Accessibility (ASSETS '13). ACM, New York, NY, USA, Article 23, 8 pages. DOI : http://dx . doi.org/10.1145/2513383.2513437

21. Scott D. Novich and David M. Eagleman. 2015. Using space and time to encode vibrotactile information: toward an estimate of the skin's achievable throughput. Experimental Brain Research 10 (2015). DOI : http://dx . doi.org/10.1007/s00221-015-4346-1

22. Jeha Ryu, Ian Oakley, Yeongmi Kim, and Jongeun Cha. 2009. A Tactile Glove Design and Authoring System for Immersive Multimedia. IEEE MultiMedia 17, undefined (2009), 34-44. DOI : http://dx . doi .org/ doi . ieeecomputersociety .org/10.1109/MMUL . 2009.63

23. Oliver Schneider, Siyan Zhao, and Ali Israr. 2015. FeelCraft: User-Crafted Tactile Content. Springer Japan, Tokyo, 253-259. DOI : http://dx . doi .org/10.1007/978-4-431-55690-9_47

24. Caitlyn Seim, James Hallam, Shashank Raghu, Tri-An Le, Greg Bishop, and Thad Starner. 2015. Perception in Hand-Worn Haptics: Placement, Simultaneous Stimuli, and Vibration Motor Comparisons. Technical Report. Georgia Institute of Technology.

25. Caitlyn E. Seim, David Quigley, and Thad E. Starner. 2014. Passive Haptic Learning of Typing Skills Facilitated by Wearable Computers. In CHI'14 Extended Abstracts on Human Factors in Computing Systems (CHI EA '14). ACM, New York, NY, USA, 2203-2208. DOI : http://dx . doi .org/10.1145/2559206.2581329

26. C. Spence and C. Ho. 2008. Tactile and Multisensory Spatial Warning Signals for Drivers. Haptics, IEEE Transactions on 1, 2 (July 2008), 121-129. DOI : http://dx . doi . org/10 . 1109/TOH . 2008 . 14

27. Ian R Summers, Jon J Whybrow, Denise A Gratton, Peter Milnes, Brian H Brown, and John C Stevens. 2005. Tactile information transfer: A comparison of two stimulation sites. The Journal of the Acoustical Society of America 118, 4 (2005), 2527-2534.

28. C. Swindells, S. Pietarinen, and A. Viitanen. 2014. Medium fidelity rapid prototyping of vibrotactile haptic, audio and video effects. In 2014 IEEE Haptics Symposium (HAPTICS). 515-521. DOI : http://dx.doi .org/10.1109/HAPTICS. 2014.6775509

29. David Ternes and K E MacLean. 2008. Designing Large Sets of Haptic Icons with Rhythm. In EuroHaptics, M. Ferre (Ed.). Springer, Madrid, Spain, 199-208. http://www.cs.ubc.ca/labs/spin/ publications/spin/ternes2008eh.pdf

30. Francisco Vega-Bermudez and Kenneth O Johnson. 2001. Differences in spatial acuity between digits. Neurology 56, 10 (2001), 1389-1391.

31. Sabine Webel, Uli Bockholt, Timo Engelke, Nirit Gavish, Manuel Olbrich, and Carsten Preusche. 2013. An augmented reality training platform for assembly and maintenance skills. Robotics and Autonomous Systems 61, 4 (2013), 398 - 403. D0I : http://dx .doi.org/10.1016/j .robot.2012.09.013 Models and Technologies for Multi-modal Skill Training.

32. Benjamin W. White, Frank A. Saunders, Lawrence Scadden, Paul Bach-Y-Rita, and Carter C. Collins. 1970. Seeing with the skin. Perception \& Psychophysics 7, 1 (1970), 23-27. DOI : http://dx.doi.org/10.3758/BF03210126

33. Cheng Xu, Ali Israr, Ivan Poupyrev, Olivier Bau, and Chris Harrison. 2011. Tactile Display for the Visually Impaired Using TeslaTouch. In CHI'11 Extended Abstracts on Human Factors in Computing Systems (CHI EA '11). ACM, New York, NY, USA, 317-322. DOI : http://dx.doi.org/10.1145/1979742.1979705

34. Nesra Yannier, Ali Israr, Jill Fain Lehman, and Roberta L. Klatzky. 2015. FeelSleeve: Haptic Feedback to Enhance Early Reading. In Proceedings of the 33rd Annual ACM Conference on Human Factors in Computing Systems, CHI 2015, 2015. 1015-1024. DOI : http://dx . doi .org/10.1145/2702123.2702396 